\title{
L'apparition des sociétés immobilières et les mutations du marché architectural : l'exemple lausannois (1860-1880)
}

Dave Lüthi

\section{CpenEdition}

Journals

Édition électronique

URL : http://journals.openedition.org/edl/236

DOI : $10.4000 /$ edl.236

ISSN : 2296-5084

Éditeur

Université de Lausanne

\section{Édition imprimée}

Date de publication : 15 décembre 2010

Pagination : 125-154

ISBN : 978-2-940331-24-6

ISSN : 0014-2026

\section{Référence électronique}

Dave Lüthi, « L'apparition des sociétés immobilières et les mutations du marché architectural :

l'exemple lausannois (1860-1880) », Études de lettres [En ligne], 4 | 2010, mis en ligne le 15 décembre

2013, consulté le 19 décembre 2020. URL : http://journals.openedition.org/edl/236 ; DOI : https:// doi.org/10.4000/edl.236 


\title{
L'APPARITION DES SOCIÉTÉS IMMOBILIÈRES ET LES MUTATIONS DU MARCHÉ ARCHITECTURAL: L'EXEMPLE LAUSANNOIS (1860-1880)
}

\begin{abstract}
Dans le deuxième tiers du XIXe siècle, les sociétés commerciales s'emparent du secteur constructif, lui insufflant un renouveau formel et fonctionnel. Grâce à des moyens financiers accrus, les édifices ou les ensembles bâtis présentent une monumentalité inconnue auparavant; en outre, édifiés en vue de promotion immobilière ou de location, ils n'abritent plus les maîtres de l'ouvrage et doivent donc être conçus pour un "client type». Une véritable mutation des habitudes architecturales s'en suit. L'exemple lausannois est très représentatif puisque la ville doit son expansion presque exclusivement à des sociétés privées, les autorités libérales se refusant à prendre en charge l'urbanisation d'un chef-lieu alors en plein essor.
\end{abstract}

Durant la deuxième moitié du XIX ${ }^{\mathrm{e}}$ siècle, les conditions de la commande architecturale évoluent de manière remarquable. On constate un véritable phénomène de démocratisation de la construction; le recours à un architecte n'est plus réservé à une élite, mais il se répand bien plus largement dans la société. La production bâtie qui en résulte, longtemps négligée parce que perçue comme une production de série, a été sensiblement réhabilitée dès les années 1980; elle demeure malgré tout méconnue et peu étudiée. Se positionner du côté du maître de l'ouvrage est un moyen sans doute pertinent, bien qu'en grande partie inédit, d'en comprendre les tenants et les aboutissants. A y regarder de plus près, la typologie des "commanditaires" de cette période apparaît en effet beaucoup moins homogène qu'aux époques antérieures. Aux individus s'ajoutent les sociétés commerciales (anonymes, immobilières) qui vont jouer un rôle déterminant dans la mutation des pratiques de la commande et de la création architecturale, mais aussi de 
la fonction même des édifices, dans les années 1850-1880 en particulier. Des exemples lausannois documentés par des sources jamais exploitées encore ${ }^{1}$ permettent la mise en évidence du rôle majeur des sociétés dans l'essor urbain. Ces associations privées agissent non seulement sur le marché immobilier qu'elles contribuent à étoffer, mais aussi sur la forme même de la ville. Abandonnant la formule traditionnelle de l'immeuble de rapport de petit format, elles réalisent des rues voire des quartiers entiers. Elles imposent la vision urbaine d'une certaine classe sociale à une population large mais aussi à ses représentants, les autorités communales, qui se singularisent alors par une passivité tout à l'avantage des promoteurs privés. De qui sont constituées ces sociétés, dans quels buts agissent-elles? En quoi la production architecturale qui est la leur rompt-elle avec les usages traditionnels? Autant de questions auxquelles quelques études de cas chercheront à répondre et à thématiser.

\section{Qui est client en $1850 ?$}

Selon les registres de l'assurance-incendie, neuf édifices seulement sont bâtis, agrandis ou reconstruits durant les années 1850 à Lausanne. Cette proportion très restreinte illustre la somnolence qui singularise le marché d'alors. Les plus prestigieux de ces chantiers sont dus à des membres influents de la politique ou du commerce comme le conseiller d'Etat Henri Fischer, les notables Charles Auguste Dapples, MarcAuguste Crausaz, Sigismond Charles de Charrière, et le banquier Charles Bugnion. Dans quelques cas, il s'agit de résidences périurbaines, les fameuses "campagnes" (Le Solitaire pour Fischer, La Grande Vuachère pour Dapples, L'Hermitage pour Bugnion); on a sinon affaire à des demeures urbaines. Mais de façon générale, toutes sont destinées au propre logement des maîtres de l'ouvrage et, parfois, à celui de locataires, ainsi que le laisse penser certaines descriptions des assureurs: "Bâtiment neuf excavé, huit compartiments de caves voûtées; trois magasins et arrière-magasins, chambre et cuisine; six pièces et cuisine; $2^{\mathrm{e}}$ et $3^{\mathrm{e}}$ idem; mansardes: huit pièces et cuisine et étendage, couvert

I. Ces sources sont notamment: ACV, K XV L, polices de l'assurance-incendie, 1850-1891; AVL, RE 17/5-11, registres de la Direction des travaux de la ville, $1857-$ 1882. 
en tuiles» relève-t-on pour l'immeuble construit peu avant 1857 pour Georges Moïse Marc Louis Meylan au Grand-Saint-Jean, visiblement destiné à être habité par plusieurs ménages en plus de lui-même ${ }^{2}$.

De cette radiographie très sommaire, on tire quelques enseignements. Il apparaît avec clarté que le champ de la construction est réservé à la classe aisée et que celle-ci investit dans l'immobilier généralement pour son propre compte. Les commerçants ajoutent aux immeubles des boutiques destinées à leur usage, ainsi que des appartements à donner en location; par ce biais, ils augmentent leur gabarit - aucun règlement n'est là pour fixer de limites - et leur rendement financier. Les dimensions des immeubles de rapport de cette époque restent pourtant très modestes: quatre niveaux en général, et quatre ou cinq rangées de fenêtres en façade au maximum. On peut voir dans cette situation quelque peu étouffée la conséquence directe du système politique vaudois d'avant la révolution libérale de 1830: comme le rappelle Gérald Arlettaz, pour être électeur (et donc éligible), il faut être propriétaire foncier et «appartenir aux trois quarts des citoyens les plus imposés de la commune» ${ }^{3}$. Seule une partie infime des citoyens obtient ce droit (entre $10 \%$ et $27 \%$ en moyenne, sans doute moins de $10 \%$ pour la ville de Lausanne); la chose politique reste une affaire oligarchique pour laquelle la propriété joue un rôle central. Les bouleversements politiques de 1830 vont modifier cette conception restrictive en réformant d'abord le droit électoral; mais il faudra attendre la révolution suivante, celle de 1845, pour voir la fondation d'une banque cantonale par les radicaux qui sert avant tout à financer les investissements fonciers et immobiliers - ce qui empêche de fait tout retour au système inégalitaire précédent. Dès lors, construire ne revêt plus le même sens: d'un signe de domination à caractère politique et social, constituant aussi un patrimoine au sens financier du terme, l'acte d'édifier se mue peu à peu en une représentation de la puissance économique et comme un placement rentable.

Dans un premier temps, le rapport du maître de l'ouvrage à l'architecte ne se modifie guère. Celui-ci travaille toujours pour un particulier qui lui commande des plans et lui confie la construction de la demeure - tâche qui n'est guère encore déléguée à un entrepreneur,

2. A l'emplacement de l'actuel 12, rue Pichard. ACV, K XV L, Lausanne, 1857, vol. 5 , f ${ }^{\circ} 9$.

3. G. Arlettaz, Libéralisme et société dans le canton de Vaud, p. 111 sq. 
l'architecte endossant généralement ces deux rôles. Le rapport commercial est clair: un pourvoyeur de service travaille pour un commanditaire, il répond à ses besoins, définis dans la plupart des cas en commun. En clair, le client établit un programme que l'architecte transcrit en termes architecturaux en tenant compte d'une enveloppe économique donnée. Le commanditaire fait ensuite modifier le projet si besoin est, pour des raisons souvent financières, plus rarement esthétiques. L'exemple du couple Gabriel et Anna Eynard illustre bien le rapport dialectique entre le créateur et les maîtres de l'ouvrage ${ }^{4}$, même si dans le cas précis, ces derniers possèdent des connaissances artistiques qui dépassent la moyenne habituelle. Les architectes se retrouvent à la merci de leurs clients et leurs projets souvent s'en ressentent: un appauvrissement des formes résulte généralement de ce jeu de va-et-vient. La seule exception à ce schéma intervient lorsque l'architecte se substitue à son client et qu'il édifie une demeure pour son propre usage. A Lausanne, on peut mentionner à ce propos le pavillon d'Henri Perregaux en Mornex (1829-1832, agrandi en 1833-1834), Mi-Mont, résidence élevée par l'ingénieur cantonal Adrien Pichard pour lui-même, Riant-Clos par Louis Wenger (1847) ${ }^{5}$ et, plus tardivement (1877), la double maison construite conjointement à la rue Neuve par les deux inspecteurs des bâtiments de l'Etat François Sudheimer et Paul Charton. Dans tous ces exemples, les édifices se signalent comme des faire-valoir de la pratique personnelle et constituent de véritables chefs-d'œuvre pour chacun de ces architectes; un seul subsiste hélas à ce jour (fig. 1).

\section{Un nouveau client: la société immobilière}

En raison de l'essor économique et démographique de la ville provoqué par la politique du nouveau régime radical (17 108 habitants en 1850, 25845 en 1870, 46732 en 1900) ${ }^{6}$, le parc immobilier traditionnel ne suffit plus à loger les nombreux travailleurs qui émigrent dans le chef-lieu, pas plus que la bourgeoise dont les attentes en matière d'habitat évoluent

4. A. Corboz, «Le palais Eynard à Genève»; P. Bissegger, Entre Arcadie et Panthéon.

5. M. Grandjean, Les Monuments d'art et d'histoire du canton de Vaud, p. 14, 77 sq. et 82 .

6. Chiffres du Bureau fédéral des statistiques, publiés in INSA, p. 240. 


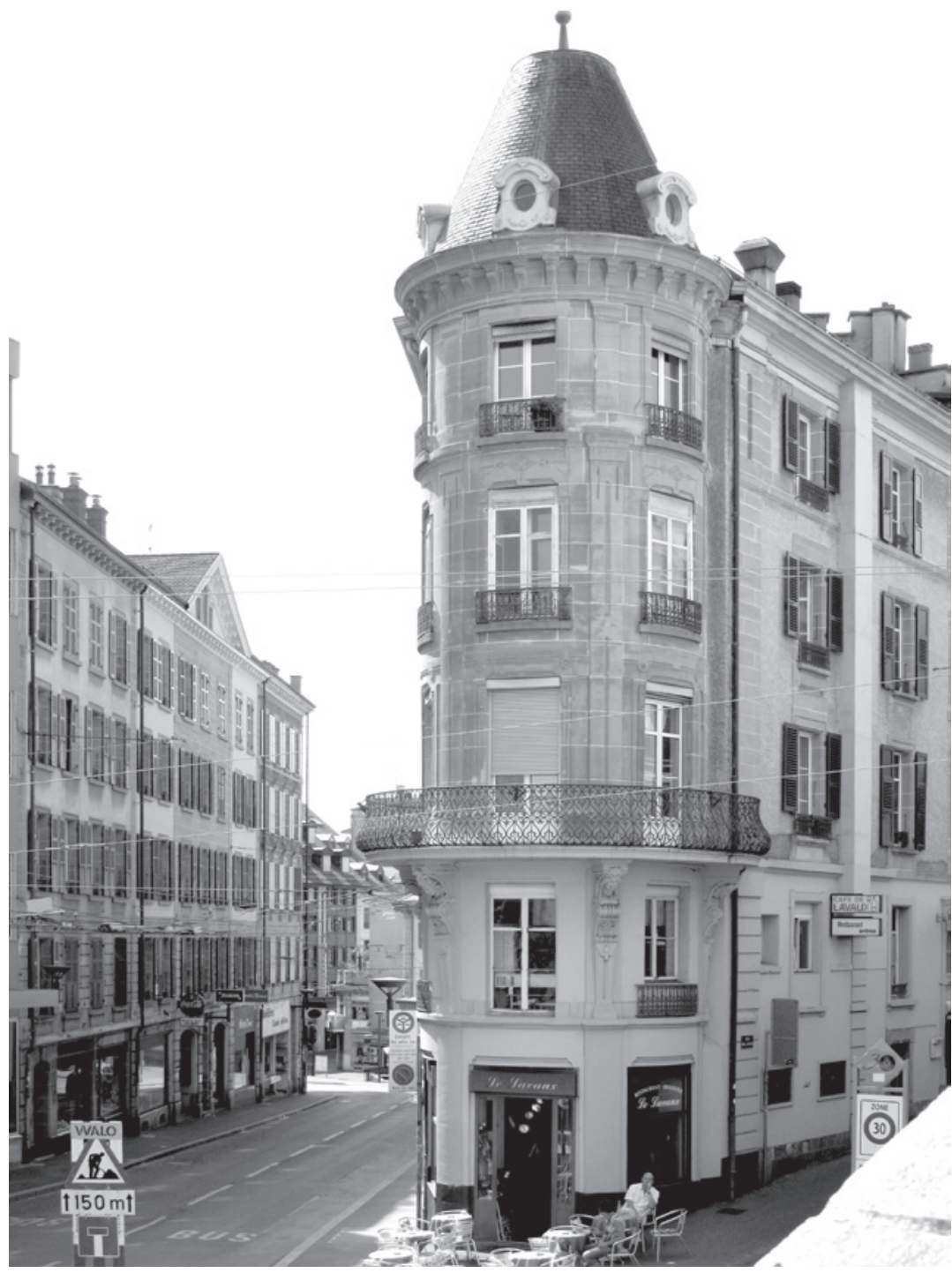

Fig. 1 - Lausanne, rue Neuve, immeuble édifié en 1877 par les deux architectes François Sudheimer et Paul Charton. 


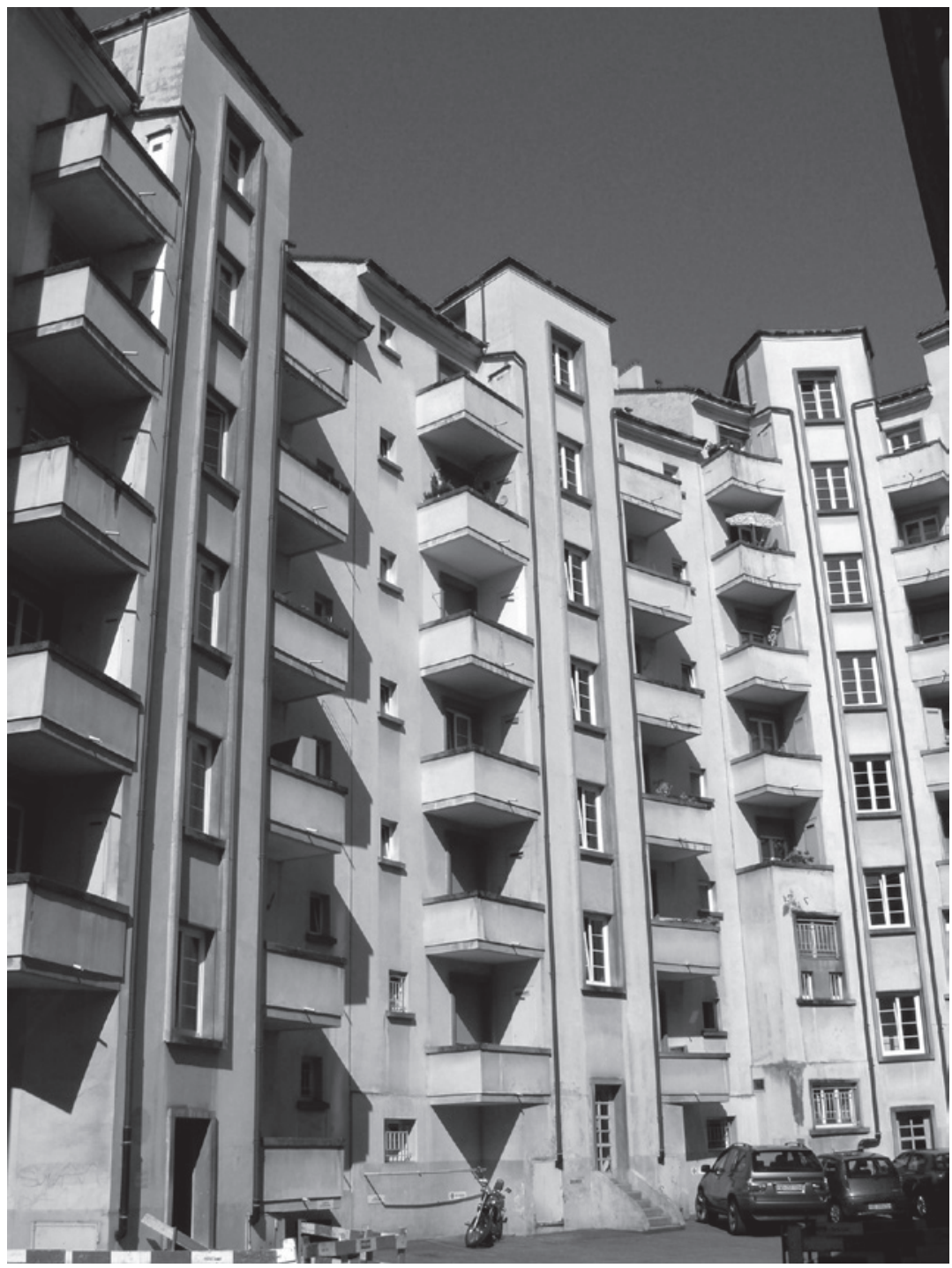

Fig. 2 - Lausanne, immeubles à l'angle des rues Marterey et Sainte-Beuve. Bel exemple de promotion immobilière des années 1930 qui se solde par la faillite de la société responsable de la construction, faute de locataires. 
considérablement. En dépit de la Grande dépression des années 18751895, le marché augmente et la ville croît à un rythme soutenu. Certes, l'apothéose de ce boom sera plus tardive; il culminera une première fois au tournant du siècle et une seconde au début des années 1930, avant que la crise immobilière qu'il engendre ne brise ce marché trop prometteur (fig. 2). Même si elles sont plus modestes quantitativement parlant, les années 1870-1880 seront décisives; de nombreux grands ensembles urbains lausannois datent en effet de cette époque ${ }^{7}$. La production de cette période demeure cependant peu étudiée en raison de la rareté des sources documentaires, mais aussi parce qu'elle a en grande partie disparu, sa position périphérique en faisant la victime de nouvelles étapes de densifications urbaines entreprises dès la fin du XIX ${ }^{\mathrm{e}}$ siècle déjà ${ }^{8}$.

Une telle mutation de la demande et de l'offre ne peut s'opérer que grâce aux prêts accordés non seulement par la Banque cantonale mais aussi par la Caisse hypothécaire d'amortissement, sa succursale fondée en 1858 par le Grand Conseil. La logique économique qui prévaut dès lors - et notamment depuis la nouvelle loi cantonale sur les sociétés commerciales votée en 1852 - implique une modification majeure du domaine immobilier. En effet, ses acteurs ne sont plus uniquement des maîtres de l'ouvrage individuels, des "personnes physiques" pour reprendre le vocable juridique, mais de plus en plus fréquemment des sociétés anonymes, des "personnes morales" donc, constituées de plusieurs membres mettant en commun des financements privés ou empruntés. Selon la loi de 1852, ce type de société, qui «ne peut exister qu'avec l'autorisation du Conseil d'Etat» (art. 23), «est qualifi[é] par la désignation de l'objet de son entreprise" (art. 16) et les associés qui la gèrent "ne sont passibles que de la perte du montant de leur intérêt dans la société» (art. 19) 9. Dans les faits, ces promoteurs (chefs d'entreprises, banquiers, notables et philanthropes) sont mus par des motifs disparates et à priori contradictoires; ainsi, ils peuvent se regrouper dans le but de

7. Outre les exemples que nous allons commenter, citons les rues Beau-Séjour et du Midi, l'ensemble de Georgette (squares et villas), le quartier du Servan, les BellesRoches, etc. A leur sujet: INSA.

8. Nous avons esquissé une première analyse de ce patrimoine fantôme dans une étude non publiée: D. Lüthi, Le quartier du Mont-d'Or à Lausanne.

9. Loi du 14 décembre 1852 sur les sociétés commerciales, in Recueil des lois, décrets et autres actes du Gouvernement du Canton de Vaud, et des actes de l'Assemblée fédérale et du Conseil fédéral de la Confédération suisse, p. 385-397. 
fournir un logement décent à une population souvent miséreuse ou, au contraire, en vue d'édifier des immeubles luxueux à donner en location ou en vente à la classe aisée. Cette seconde option semble mieux s'accorder avec les idéaux capitalistes des promoteurs immobiliers. Il ne faut toutefois pas voir une opposition de principe entre l'action philanthropique de certaines sociétés et l'attente d'un rendement, voire d'un bénéfice. Des penseurs protestants tels que le pasteur vaudois Charles Archinard ont en effet postulé que l'intérêt général se nourrit de l'intérêt individuel et que seule la croissance économique et démographique peut engendrer une augmentation de la richesse d'un pays. Le bonheur des individus dépend donc du développement de l'industrie, qui a un but moral et social à la fois ${ }^{10}$. La bourgeoisie lausannoise peut donc investir dans la pierre en toute bonne conscience, puisque, d'une manière ou d'une autre, elle contribue au bien économique du pays ${ }^{11}$.

\section{Les sociétés à but philanthropique}

L'une des plus anciennes sociétés anonymes lausannoises a justement un but philanthropique déclaré. Elle résulte de la réunion en 1861 d'une cinquantaine de citoyens «en vue de [...] construire ou approprier des logements pour les familles d'ouvriers " ${ }^{12}$. Sous le nom de Société de construction à Lausanne, elle va ériger plusieurs immeubles d'habitation populaires: trois à la place du Tunnel (1862-1864) et cinq autres au Vallon et à la rue du Nord (1871-1876) (fig. 3) ${ }^{13}$. Toutefois, comme le stipulent ses statuts, si elle a "pour objet essentiel la construction, la vente, et, au besoin, l'administration de maisons pour la classe ouvrière", elle ne doit pas «s'interdire [...] d'autres constructions et opérations

Io. Ch. Archinard, Le Canton de Vaud et l'industrie; à son sujet, G. Arlettaz, Libéralisme et société dans le canton de Vaud, p. 169 sq.

II. Notons cependant que plusieurs ensembles urbains de haut standing sont réalisés par des sociétés simples, regroupant des propriétaires fortunés (rues du Midi et de BeauSéjour notamment) (AVL, 221/11/4/1 sur la construction de la rue du Midi, 1871-1874).

I2. Société de construction à Lausanne.

I3. Cet ensemble a été étudié de manière approfondie par G. Barbey, J. Gubler et A. Abriani, "Exemples d'habitations ouvrières au tournant du siècle", p. 318-320; voir aussi G. Heller, "Propre en ordre", p. 81-84; M. Grandjean, Les Monuments d'art et d'histoire du canton de Vaud, p. 185 sq. 


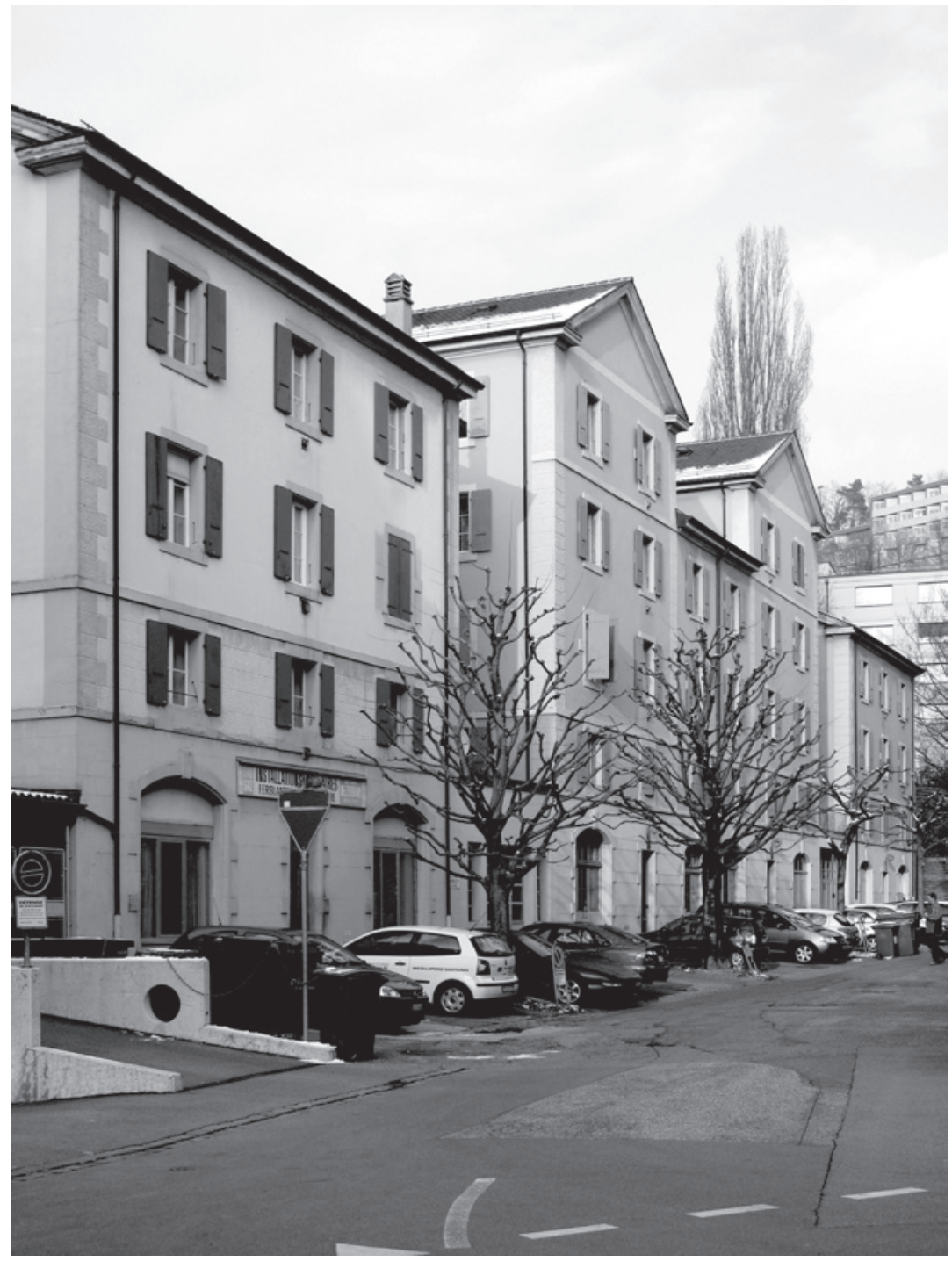

Fig. 3 - Lausanne, rue du Nord, immeubles édifiés par la Société de construction au milieu des années 1870 . 
rentrant dans le cercle naturel de son activité et pouvant lui faciliter l'accomplissement de son but principal, qui est d'offrir aux familles d'artisans et ouvriers des logements suffisants, propres, salubres et d'un prix modéré» ${ }^{14}$. Ainsi, en "faisant une œuvre philanthropique, la Société de construction [ne veut pas] faire l'aumône à ses locataires. Ses règlements statuent que les sommes versées produiront intérêt à raison de quatre pour cent. Les bénéfices, s'il y en a, se répartissent de la manière suivante: $40 \%$ sont distribués aux actionnaires à titre de dividende en sus de l'intérêt; $20 \%$ sont partagés entre le gérant et les employés de la Société; $40 \%$ sont appliqués à former un fonds de réserve» ${ }^{15}$. On notera que l'intérêt de $4 \%$ promis aux actionnaires est exactement équivalent à celui des caisses hypothécaires du temps ${ }^{16}$. En véritable entreprise commerciale, la Société va user de ses droits et se défaire de ses immeubles du Tunnel pour financer ceux du Vallon, en même temps qu'elle augmente son capital-actions avant de le réduire à nouveau ${ }^{17}$. En tout, la Société aura érigé au Vallon et au Nord 8 bâtiments, 24 magasins ou ateliers et 74 appartements, au Tunnel 34 magasins et 130 appartements.

Ses réalisations se caractérisent par leur extrême rigueur formelle. Les édifices sont assez mal situés (ceux du Tunnel, situés en contrebas de la rue de l'Université, donnent en grande partie au nord-ouest et ceux du Vallon, bien que tournés au sud, se situent dans une vallée humide, peu ensoleillée et dévolue à l'industrie), les appartements sont très restreints et leur confort, sommaire. Les auteurs de leurs plans ne sont pas connus, mais il s'agit vraisemblablement des architectes Achille de La Harpe et Jean-Baptiste Bertolini ${ }^{18}$ pour les immeubles du Tunnel, et peut-être d'Ernest Dapples, ingénieur ferroviaire et membre du comité, pour ceux du Vallon. En effet, l'élévation des façades sur la rue du Nord, à «l'articulation néoclassique et [aux] combles croisés “à la Durand" " 19

14. Statuts de la Société de construction à Lausanne.

15. "Les habitations ouvrières - Isolement ou casernement", p. 63.

I6. La Caisse hypothécaire d'amortissement offre ce taux en 1860 (Crédit foncier vaudois, p. 29).

I7. Celui-ci se monte à 200000 fr. en 1861, 200 actions; 400000 fr. en 1872, 400 actions; 354500 fr. en 1886, 709 actions.

I8. AVL, RE $17 / 6,1^{\text {er }}$ juillet 1863 , p. 64 ; discussion entre ces architectes et la Direction des travaux au sujet des constructions en cours au Tunnel. Ils ne sont plus cités par après.

19. INSA, p. 348. 
peut apparaître comme le produit d'une architecture d'ingénieur, de même que la standardisation du programme et la systématisation des plans qui montrent une économie évidente des moyens. Les éléments du confort se réduisent à l'éclairage au gaz des cages d'escaliers (très sombres par ailleurs) et, pour une maison seulement, à l'eau courante ${ }^{20}$. A ce sujet, "l'expérience faite a engagé à ne pas continuer [cet] essai. Outre les ruptures de canalisation par le gel, ou autres causes, [on] craint les accidents pouvant résulter de la légèreté des nombreux enfants qui occupent ces appartements. Il suffit d'un robinet ouvert par mégarde ou par enfantillage pour causer de grave dégâts ${ }^{21}$.

La question de la typologie du logement ouvrier, qui est alors un enjeu majeur de ce type de construction ${ }^{22}$, a été longuement étudiée par la Société. Ses membres hésitent entre le type de la caserne ouvrière et celui, beaucoup plus en vogue, du petit immeuble à deux ou quatre logements. Le choix de la première est expliqué pour des raisons inhérentes au contexte local:

A Lausanne, l'ouvrier tient à ne pas demeurer trop loin de son centre d'activité. Et à Lausanne ou aux environs immédiats le prix du sol est tel que le capital engagé ne peut obtenir sa juste rémunération qu'en utilisant le sol autant que possible et en répartissant sur un grand nombre de loyers le prix d'achat du terrain. C'est regrettable, mais obligatoire ${ }^{23}$.

Ainsi, sous couvert de la prise en compte d'un souhait soi-disant émis par les futurs locataires, la Société privilégie la solution qui lui permet d'obtenir une «juste rémunération». Ce faisant, elle impose un style de vie très précaire aux habitants - la hauteur des loyers leur imposant souvent de cohabiter à plusieurs dans de très petites surfaces ${ }^{24}$ - et un

20. Cet élément de confort est peut-être le fait de Dapples, qui est justement spécialisé en questions hydrauliques. Outre son rôle dans la création des bains d'Ouchy, il publie des textes à ce propos (E. Dapples, Etude sur l'application des forces hydrauliques à l'exploitation des chemins de fer des montagnes et en particulier sur les chemins de fer pneumatiques).

2I. Société de construction à Lausanne, p. 11.

22. A ce propos, voir B. Corthésy, "L'ouvrier d'aujourd'hui aime aussi le confort et les belles choses." ".

23. Société de construction à Lausanne, p. 8.

24. Le recensement fédéral de 1888 fait apparaître qu'au Vallon, l'on dénombre 2,1 occupant par pièce en moyenne, contre 1,05 pour l'ensemble de Lausanne 
confort des plus élémentaires, gardant une ligne théorique proche des idées d'Archinard. Elle n'a guère de scrupule à demander en contrepartie de logements sommaires non seulement un loyer mais également l'acclimatation à un mode de vie, ainsi qu'à des valeurs bourgeoises: respects des biens d'autrui, entretien régulier, discrétion, surveillance et éducation des enfants, etc. La philanthropie n'est donc jamais éloignée du paternalisme, ni du profit.

Notons au passage que les sociétés coopératives qui apparaissent au même moment ont un fonctionnement différent puisqu'elles impliquent la participation active et financière des habitants et, de fait, la production architecturale qu'elles engendrent est tout autre. L'Association coopérative immobilière de Lausanne, formée en 1869, a ainsi pour but la "construction d'habitations suivant des prix et des modèles déterminés, présentant de bonnes conditions de salubrité, avec jardins et destinées essentiellement à une seule famille " ${ }^{25}$. Il ne s'agit pas encore, dans ce cas, d'une coopérative mutualiste comme il s'en créera à la fin du siècle, mais d'une société philanthropique qui cherche à «faciliter au locataire l'acquisition de son habitation par voie d'amortissement» ${ }^{26}$. Elle prône notamment, par le biais de maisons individuelles munies d'un jardin, "la vie au grand air si favorable à la santé des enfants et l'attachement de la famille au foyer qui lui appartient » ${ }^{27}$. L'architecte Georges Rouge, membre du comité et inspecteur des bâtiments de la Ville, a prévu une architecture modeste mais soignée, dont la "décoration extérieure a été obtenue par la disposition symétrique des pignons des maisons des ailes et par quelques ornements en bois découpés, [qui donnent] à l'ensemble un aspect attrayant ${ }^{28}$. La beauté apparaît comme un agent de l'attachement des habitants à leur foyer; la qualité de l'architecture n'est donc plus, dans ce cas, accessoire. La forme coopérative ne sera cependant pas couronnée de succès et seule une petite portion du projet de quartier prévu à la Pontaise sera réalisée (fig. 4).

(G. Barbey, J. Gubler et A. Abriani, «Exemples d'habitations ouvrières au tournant du siècle», p. 318).

25. Statuts de l'association coopérative immobilière de Lausanne.

26. Notice sur les constructions exécutées à la Ponthaise par l'Association coopérative immobilière de Lausanne.

27. Ibid.

28. Ibid. 

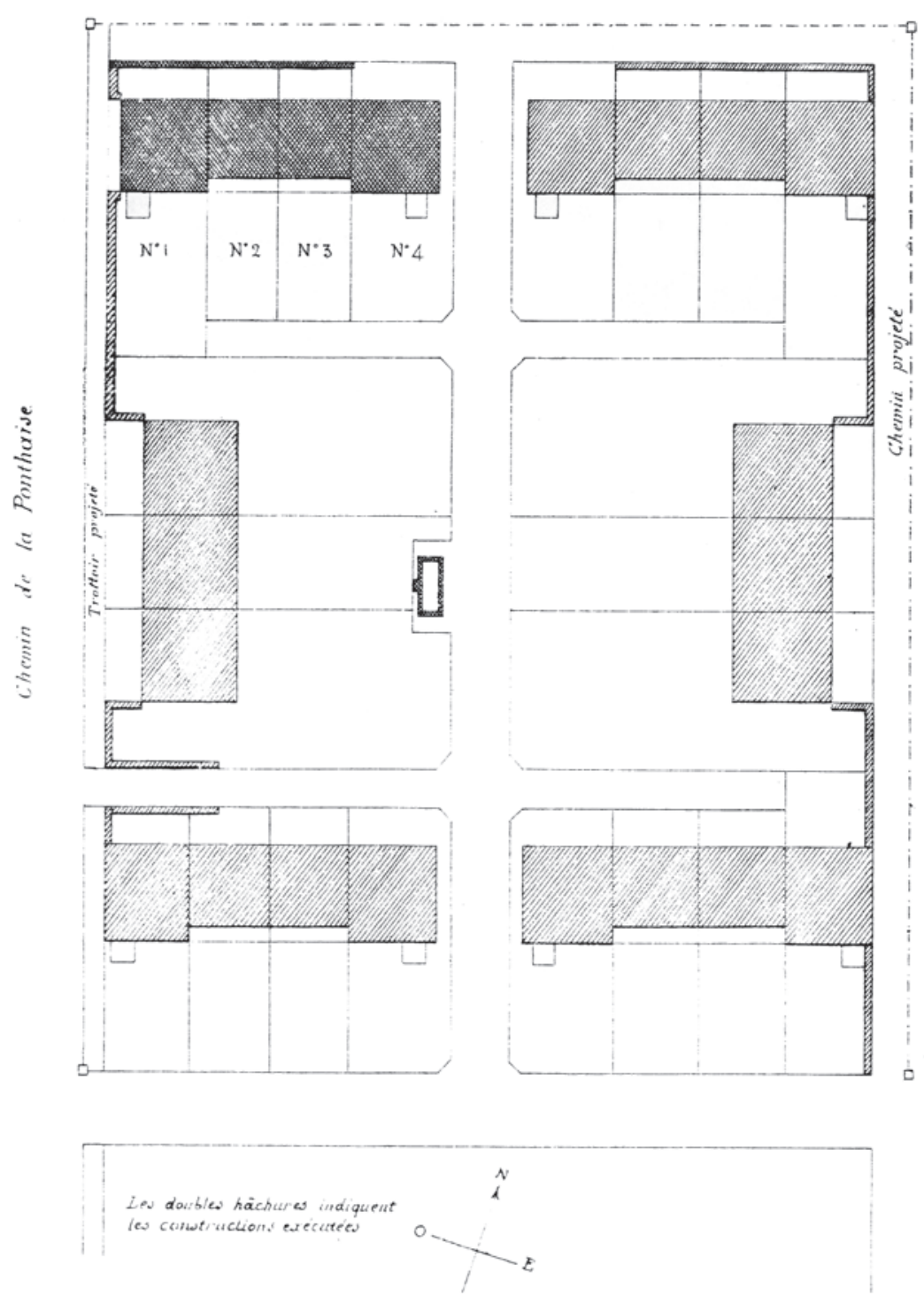

Fig. 4 - Lausanne, projet de quartier coopératif à la Pontaise, plan directeur de 1869. Seule la portion numérotée a été réalisée. (AVL). 


\section{Les sociétés immobilières}

Dès les années 1860, plusieurs sociétés immobilières dont les buts n'ont rien de philanthropique apparaissent par ailleurs. Leur objectif est clairement spéculatif ou, du moins, capitaliste: il s'agit, par des opérations immobilières, de réaliser une plus-value sur des investissements financiers souvent conséquents. La plus ancienne parvenue à notre connaissance, la Société immobilière lausannoise, est fondée par l'architecte Louis Joël en 1858 en vue de la création de la rue Haldimand et dotée d'un capital d'un million de francs ${ }^{29}$. Les relations des autorités communales sont plutôt bienveillantes à l'égard de cet ancien inspecteur des travaux de la Ville et futur syndic ${ }^{30}$. A y regarder de plus près, on constate d'ailleurs que la Société agit véritablement en remplacement des autorités pour achever la ceinture Pichard, le «Ring» lausannois. Par un décret du Grand Conseil de 1836, cette tâche lui était en effet dévolue mais pour le dernier secteur à créer, la liaison Riponne-Grand Pont, elle s'en décharge donc sur une société privée. Cette situation étonnante à nos yeux est explicitée par la commission créée par le Conseil communal pour étudier le projet soumis par Joël:

[...] en présence du développement que promet à notre Ville le réseau des chemins de fer qui y aboutissent, et vis-à-vis du mouvement industriel qui s'y fait déjà remarquer et qui tend à prendre de plus en plus d'extension [il] paraît évident que, dans de telles circonstances, et avec les besoins incessants de nouveaux logements, la création d'un quartier neuf n'est point une chose indifférente. Et comme une société immobilière lausannoise ne peut manquer de se constituer, l'établissement de cette rue neuve procurera à nos capitalistes une occasion favorable de placer leurs fonds d'une manière aussi solide qu'avantageuse, tout en offrant à la classe nombreuse des industriels, artisans et ouvriers qui nous entourent, celle de trouver une série d'occupations lucratives de longue haleine ${ }^{31}$.

29. Gazette de Lausanne, 7 février 1859.

30. A son sujet, voir ci-dessus la contribution de Gilles Prod'hom.

3I. Rapport au Conseil communal par la commission nommée le 6 décembre 1858 (Henri Henninger, Louis Gaudin, François Vallotton, Justin-Louis Guex et Maget, municipal) (AVL, 221/12/1/23). 
A aucun moment les autorités ne semblent se soucier de ces travaux urbains et la Société - dont la commission semble avoir comme par hasard anticipé le nom - joue donc un rôle majeur. La ville se chargera tout de même de la construction de la route proprement dite et jouera un rôle non négligeable dans les difficiles affaires d'expropriation. La plupart des travaux sont sinon entrepris par la Société qui érige - ou fait ériger ${ }^{32}$ - en 1860-1861 une grande partie des immeubles qui bordent la partie supérieure de la rue (entre la place Saint-Laurent et la Riponne) et, surtout, fait dresser son plan par Joël, mission qui semble plutôt ressortir aux services communaux ${ }^{33}$. En comparaison avec les réalisations des sociétés philanthropiques déjà citées, la différence est frappante. La rue Haldimand constitue en effet le premier percement du tissu urbain médiéval lausannois et la plus précoce des rues «modernes» aménagées dans le périmètre de l'enceinte médiévale ${ }^{34}$. Hormis la liaison entre la gare et la place Saint-François via Georgette - qui n'est pas un percement ${ }^{35}$-, la ville ne crée aucune rue nouvelle de son propre chef avant la fin du siècle. A l'époque de sa création, l'échelle urbaine de la rue Haldimand frappe donc car elle n'a pas d'antécédent; elle frappe d'autant plus qu'elle est le fait d'une société anonyme privée.

Contrairement aux exemples du Vallon et du Tunnel, le public auquel Joël destine les édifices est constitué de commerçants, de petits bourgeois, qui vont peu à peu acheter les immeubles élevés par la Société. Le chantier est un laboratoire: l'architecte y emploie "pour la première fois dans notre pays [...] des mortiers faits avec la chaux hydraulique au moyen de laquelle on obtient une dessification prompte pour les murs et une solidification immédiate de la construction » ${ }^{36}$. L'aspect général de la rue est déterminé par sa forme légèrement courbe, par les dispositifs

32. Sont attestés les entrepreneurs Georges Krieg et Louis Maget Ballenegger (AVL, 221/12/41, convention avec la ville, $1^{\text {er }}$ juillet 1861).

33. Cette science n'est toutefois pas le point fort de Joël; à la demande de la Ville, son plan d'alignement sera corrigé par l'ingénieur Victor Dériaz et l'architecte Henri Boisot, ancien inspecteur des bâtiments de la ville et de l'Etat. Ils produisent un nouveau projet, muni d'une rue plus large et dont le raccordement avec la place SaintLaurent est plus aisé (AVL, 221/12/1/5 et 6, rapport de Dériaz et Boisot; rapport de S. Maget à la Municipalité, 14 février 1858).

34. Comme les rues de la Paix et du Lion-d'Or à Saint-Pierre (1908-1914) et la rue Pichard, entre le Grand-Pont et Saint-Laurent (1912).

35. A ce sujet, INSA, p. 272-280.

36. Gazette de Lausanne, 6 mai 1862. 


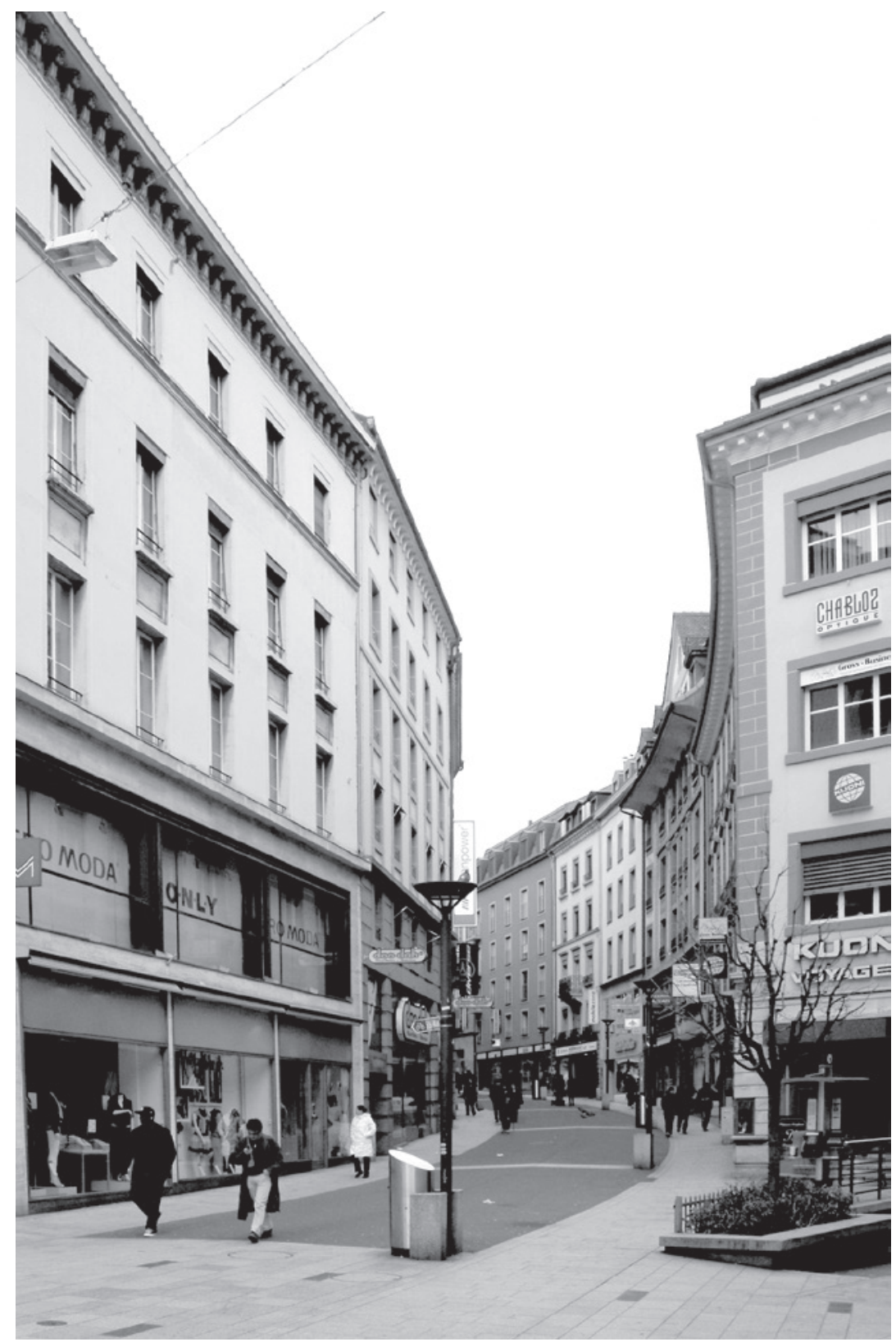

Fig. 5 - Lausanne, rue Haldimand, vue générale. 
d'entrée à pans coupés inspirés du modèle parisien, bien marqués du côté de la Riponne, et l'architecture uniforme des immeubles (fig. 5). Ceux-ci comptent un niveau de boutique, un entresol et trois étages habitables; leur façade sur rue est percée de trois ou cinq axes de fenêtres. Les devantures montrent des "vitrages [de] dimensions considérables" permise par l'usage de "gros fers pour poutres armées et de colonnes de même métal ${ }^{37}$. Elles sont traitées extérieurement dans de beaux matériaux (marbre de Saint-Triphon sans doute); la partie haute des façades est parfois ornée de balcons à garde-corps de fonte portés par des consoles de pierre sculptée. Un bandeau divise les façades à mi-hauteur, selon un procédé visible dans les grandes opérations un peu plus anciennes du Quai des Bergues à Genève (vers 1840) ou du quartier de l'hôpital à Bâle (Kaufhausgasse en particulier, 1842-1843) ${ }^{38}$; il sert à unifier visuellement l'alignement des façades, de même que les corniches à modillons. Rien de monotone cependant:

L'architecture de l'ensemble des bâtiments est simple et uniforme et cependant aucun n'est absolument semblable à son voisin. Les façades sont décorées de balcons et les fenêtres pourvues de stores d'un bon effet ${ }^{39}$.

A chaque étage sont disposés un ou deux appartements de trois à quatre pièces, assez vastes, mais conçus de manière peu hygiénique: dans leur état initial, la plupart d'entre eux montrent des chambres de bonnes et des cuisines borgnes, ainsi que de nombreuses alcôves, parfois très profondes. En raison des nouvelles normes sanitaires, certains immeubles seront transformés au début du $\mathrm{XX}^{\mathrm{e}}$ siècle afin de se défaire de ces espaces considérés comme malsains; on créera notamment des courettes d'aération et l'on supprimera les alcôves ${ }^{40}$. Toutefois, en comparaison avec les logements ouvriers, les appartements de la rue Haldimand présentent des dispositions et des dimensions qui les rendent bien plus confortables.

37. Gazette de Lausanne, 6 mai 1862.

38. A. Nagel, M. Möhle et B. Meles, Die Kunstdenkmäler des Kantons Basel-Stadt, p. 511-513.

39. Gazette de Lausanne, 6 mai 1862.

40. Voir par exemple le $n^{\circ} 11$, remanié en 1902 par l'architecte Ernest Moachon (AVL, archives de la police des constructions). 
Un autre exemple immobilier prégnant sur le territoire mérite d'être mentionné ici: il s'agit du quartier Grancy-Mont-d'Or, dû à la Société foncière des Boulevards ${ }^{41}$. Celle-ci est créée en 1872 par "quelques personnes [qui,] remarquant le développement de la ville du côté du lac, conçurent et étudièrent le projet d'acquérir des terrains situés en-dessous et le long de la voie ferrée, terrains magnifiquement exposés mais fort divisés et sans dévestitures commodes» ${ }^{42}$ (fig. 6). Le fondateur et futur président n'est autre qu'Adrien Mercier, fils du tanneur lausannois JeanJacques Mercier-Giegler et frère de Jean-Jacques Mercier-Marcel, l'un des plus importants industriels de la ville durant le dernier tiers du siècle. La valorisation des terrains sous-gare imaginée par Adrien Mercier n'est sans doute pas due au hasard: au même moment, son frère fonde la Compagnie du chemin de fer Lausanne-Ouchy dont le funiculaire inauguré en 1877 traverse et dessert justement les quartiers sous-gare ${ }^{43}$. Le capital initial est à la hauteur des ambitions: il se monte à 1800000 fr., ce qui est considérable en comparaison des sommes investies dans le logement ouvrier. Il est vrai que dans ce cas, le but du projet est très différent. Il s'agit de la constitution, par achat de nombreuses parcelles, d'un véritable quartier qu'il est ensuite question d'aménager d'un point de vue routier par un grand boulevard terminé par un rond-point monumental. Ce travail est vraisemblablement exécuté durant les années 1873-1875. La vente des parcelles régulières délimitées par cette nouvelle artère peut alors débuter; le but initial était sans doute de les écouler pièce à pièce à des propriétaires qui y feraient dresser leur demeure, la vente de la dernière parcelle devant marquer la dissolution de la société - ce qui n'arrivera que vers $1912^{44}$. Toutefois, en raison de la crise économique qui sévit alors, le projet ne semble guère susciter l'enthousiasme des Lausannois et la situation est critique jusqu'à la fin des années 1870 , notamment du côté du Mont-d'Or.

41. D. Lüthi, Le quartier du Mont-d'Or à Lausanne.

42. Société foncière des Boulevards, p. 3.

43. A ce propos, voir ci-dessus la contribution de J. Neuenschwander-Feihl.

44. L'immeuble édifié en 1911 par les architectes Vuilleumier \& Perregaux est une commande de la Société, alors en liquidation. En 1912, l'avenue de Villard reçoit son nom actuel de Mont-d'Or, sans doute au moment où la ville reprend les routes au profit du domaine public, alors que la Société s'est vraisemblablement dissoute. 


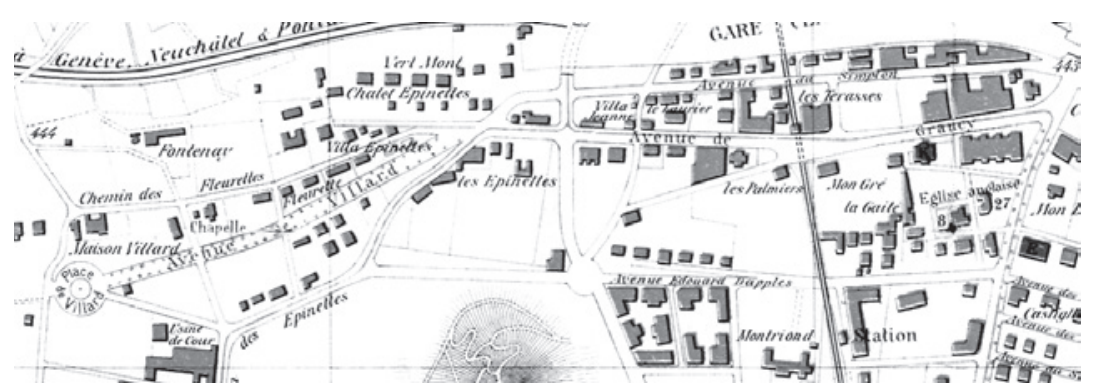

Fig. 6 - Lausanne, quartier de Grancy-Mont-d’Or, plan général (AVL).

Sans doute le quartier est-il sauvé par l'architecte-entrepreneur Gustave Conod (1833-1906) ${ }^{45}$ qui se saisit d'un grand nombre de parcelles au début des années 1880 et y fait édifier à son compte trois types de demeures: des immeubles groupés par paire à un appartement par étage, des villas mitoyennes et des villas individuelles. Il les revend ou les loue ensuite à des membres de la petite ou moyenne bourgeoisie: s'y côtoient à la fin du siècle horloger, herboriste, professeurs, ingénieurs, architecte, pasteur, rentiers, dentiste, représentants mais aussi procureur et juge fédéral ${ }^{46}$. L'architecture des demeures se prête bien à cette variété (toute partielle) des statuts sociaux. Au chemin des Fleurettes, des massifs contigus de trois niveaux contenant un appartement par étage encadrent à la manière de pavillons d'angle une rangée plus basse de villas mitoyennes de trois axes de fenêtres, coiffées d'une toiture commune en bâtière. Au nord, un jardinet marque la séparation avec la rue, alors qu'au sud un véritable jardin surélevé par rapport à l'avenue principale offre un beau dégagement à ces édifices. Hormis des chaînes d'angle lisses, les élévations sont traitées avec économie. Seuls des bandeaux et une corniche animent les façades par ailleurs sobrement percées de fenêtres rectangulaires aux encadrements de ciment (fig. 7).

Du côté du Boulevard de Grancy, c'est l'architecte-enrepreneur Gustave Wanner qui y construit le premier, à la fin des années $1880^{47}$. Ses réalisations destinées à une clientèle aisée sont beaucoup plus

45. Ce Lausannois ne doit pas être confondu avec son contemporain homonyme bernois Gustave Emile Conod (1834-1875).

46. Indicateur vaudois, années 1891 et 1900.

47. INSA donne les années 1891-1894 pour Grancy 1-7 mais selon les registres de l'assurance-incendie, ces bâtiments sont déjà terminés lors de leur taxation en 1887. 


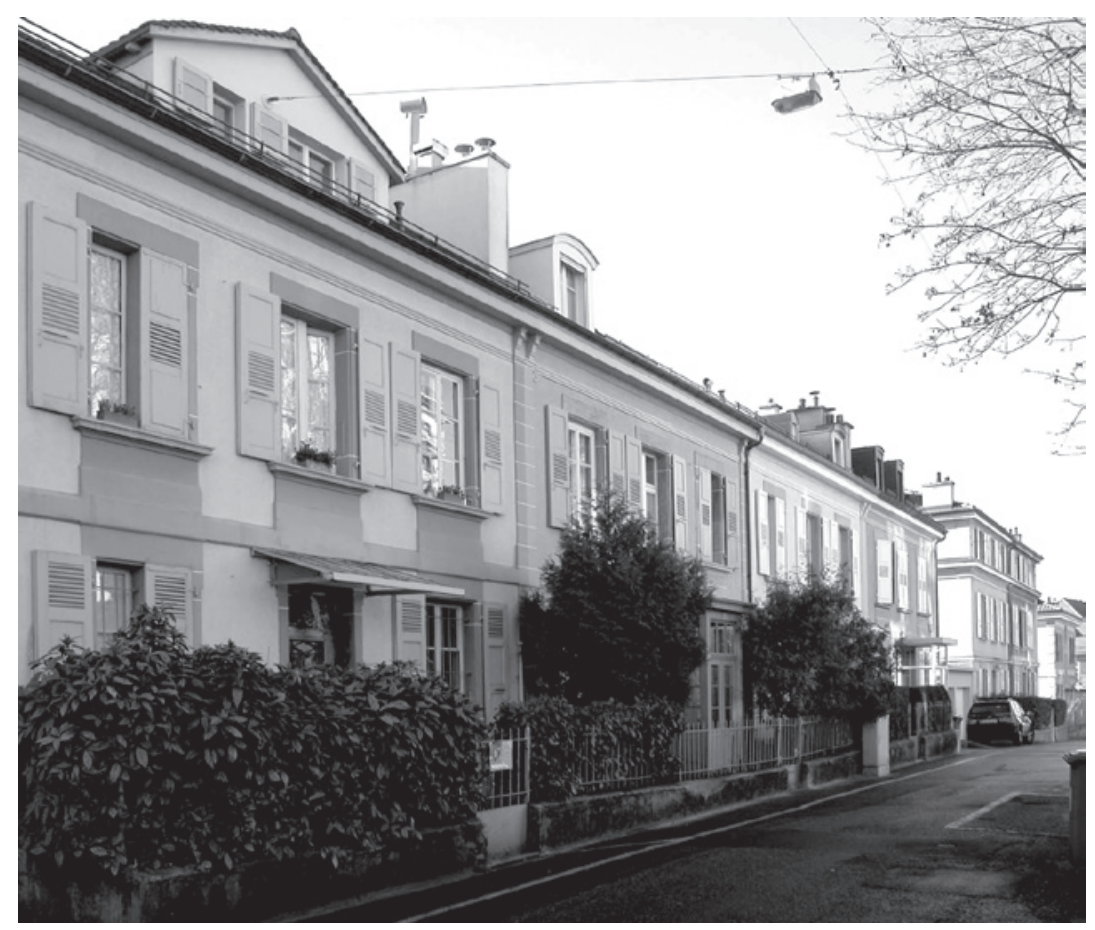

Fig. 7 - Lausanne, quartier du Mont-d'Or, façades des immeubles dus à Gustave Conod.

ambitieuses que celles de Conod et rejoignent ses immeubles antérieurs de la rue Beau-Séjour (1873) : quatre niveaux sous comble à la Mansart, un riche décor néorenaissance, des porches munis d'imposantes huisseries, des balcons à garde-corps d'une richesse alors inégalée en signalent le statut luxueux (fig. 8).

A nouveau, le recours à une Société immobilière a permis de réaliser une opération urbanistique d'envergure que la commune n'envisageait pas de mener à court terme - dans le cas précis, elle n'y pensait d'ailleurs pas du tout. Toutefois, le quartier Grancy-Mont-d'Or a ceci de particulier qu'il joue sur deux tableaux en parallèle: d'une part la Société investit pour préparer le terrain à la vente, d'autre part des particuliers y placent des fonds afin de réaliser des immeubles qu'ils vont revendre à profit, remplaçant la Société comme entrepreneurs. Elle joue donc un double rôle: si elle profite d'un retour sur investissement - certes tardivement -, elle provoque par un effet de domino la fortune d'autres acteurs 


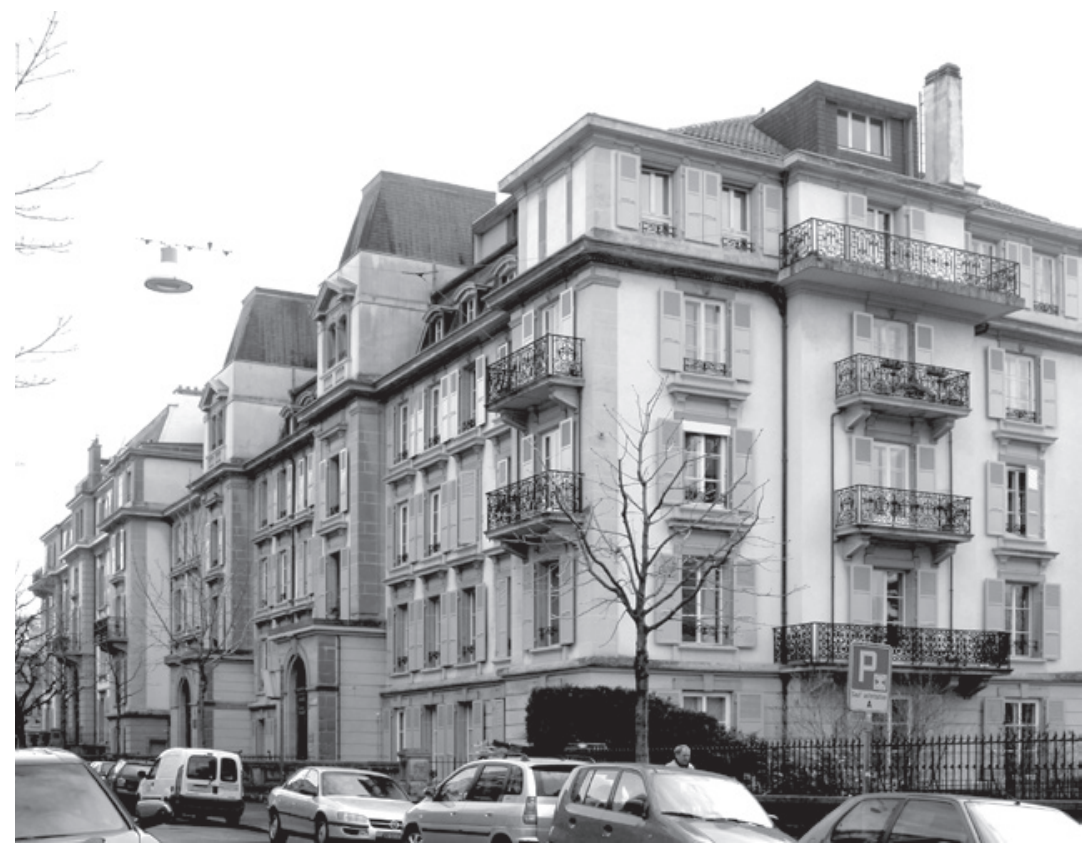

Fig. 8 - Lausanne, boulevard de Grancy, immeuble de rapport construit par Gustave Wanner.

qui lui sont parfois liés de près (à l'instar de Conod, qui est vraisemblablement un parent de l'ingénieur auteur du plan du quartier, Pellis). Sans que l'on puisse en être sûr, faute de documents à ce sujet, il semble que certaines sociétés servent de levier pour permettre la réalisation de projets architecturaux qui, isolés d'une mise en ouvre plus large, n'auraient pas pu être entrepris. La mise en commun des ressources financières permet dans ce cas surtout la réalisation des infrastructures routières et hygiéniques (égouts) ${ }^{48}$ qui demeurent possession de la Société. Elle saura d'ailleurs faire valoir cet argument auprès des autorités en cas de litige: pour preuve, la menace exercée par la Société en 1873 à propos des voies qu'elle a créées dont elle tient à informer le public de leur statut privé afin qu'il ne s'accoutume pas à les emprunter. En effet, cet usage pourrait leur être retiré si les négociations avec les autorités pour passer ces routes au

48. Du côté de Grancy, les égouts privés du quartier sont reliés à l'égout communal aménagé en 1874 lors de la réfection de l'avenue d'Ouchy par la Ville. Le raccordement est payant (AVL, Procès-verbaux de la Municipalité, 23 janvier 1874). 
domaine public (et donc éviter leur frais d'entretien) n'aboutissent pas ${ }^{49}$. Cette privatisation des voies de circulations peut paraître étonnante, mais elle se rapproche par bien des aspects des "hameaux" parisiens, ces quartiers construits par des promoteurs pour une clientèle fortunée et que clôturent aujourd'hui encore des grilles et des portails ${ }^{50}$. La situation en cul-de-sac de l'avenue de Villard à sa création est d'ailleurs très proche des impasses que forment généralement les hameaux.

\section{La société immobilière: un client impersonnel}

La transition de la commande traditionnelle entre un client "physique" et un architecte vers celle décrite ci-dessus se fait sur une période assez longue et n’apparaît pas subitement dans le marché local. Des aménagements sont sans aucun doute faits dans une pratique dont le modèle est vraisemblablement exporté des grandes villes européennes - on peut penser au système assez complexe de financement des grands travaux haussmanniens ${ }^{51}$. A aucun moment, la commande architecturale due aux sociétés ne représente un phénomène exclusif; elle cohabite toujours avec la maîtrise d'ouvrage par un client-individu, mais elle la modifie peu à peu. A partir des exemples lausannois étudiés, les caractéristiques de la société immobilière et les transformations qu'elle impose au système traditionnel peuvent être thématisées.

Dans la perspective de l'étude du client, il convient de se demander qui commande dans ce genre de configuration. De manière théorique, la société fait appel à un architecte pour lui demander un projet et elle le rétribue pour cela. Pourtant, les exemples lausannois montrent d'une part que l'architecte est parfois l'initiant du projet et que pour ce faire, il va jusqu'à fonder une société dont il sera le propre architecte; d'autre part, que lorsque la société n'est pas son propre fait, il intègre généralement son comité. Le rapport de l'architecte au projet est donc extrêmement intime, et il en maîtrise presque tous les niveaux puisque c'est à lui d'en dresser les plans et d'en assurer le financement. Son rôle dans la commande s'avère majeur; il devient son propre chef et peut déterminer

49. AVL, RB 14/49, Procès-verbaux de la Municipalité, 27 octobre 1873.

50. I. Montserrat Farguell et V. Grandval (dir.), Hameaux, villas et cités de Paris.

51. P. Pinon, Atlas du Paris haussmannien, p. 57-63 en particulier. 
sans trop de contraintes - sinon financières - l'esthétique de sa construction. Il faudrait donc reconsidérer l'architecture des édifices bâtis dans ce cadre sociétaire car peut-être représentent-ils des œuvres plus proches de la sensibilité de leur créateur que d'autres constructions dont les données de départ sont imposées par un maitre d'ouvrage ${ }^{52}$.

Le changement de nature du commanditaire est fondamental car il implique par conséquent l'émergence d'un nouvel acteur dans le jeu immobilier: l'habitant. Traditionnellement, on l'a dit, le propriétaireconstructeur occupe l'édifice qu'il fait ériger. Avec l'avènement des sociétés et de la spéculation immobilière - car c'est bien de cela qu'il s'agit - la nature du futur habitant (qu'il soit locataire ou acheteur) n'est pas connue à l'avance. Cette incertitude provoque une rupture dans la commande: ce n'est plus l'occupant qui décrit ses attentes à l'architecte, mais c'est à l'architecte de prédéfinir un programme standardisé dont on peut espérer qu'il corresponde aux besoins du plus grand nombre. Cette standardisation se décline en trois éléments au moins: le plus important est sans doute le plan, qui conditionne les deux autres, soit l'aménagement intérieur et la façade. Par des critères de surface, de distribution, de nombre et de fonctions des pièces, le premier satisfait aux besoins précis d'une classe sociale et, par conséquent, il doit tenir compte de ses conventions: le problème est particulièrement aigu pour la moyenne et la haute bourgeoisie pour lesquelles la convenance de la distribution et du décor revêt une signification particulière ${ }^{53}$. Dans le domaine de l'habitat populaire, le plan a aussi son importance puisque c'est par son biais que les philanthropes qui bâtissent les casernes peuvent imposer le contrôle social qui les obsède. Mais de part et d'autre, on peut relever l'existence de certains plans-type, dont certains remontent à des périodes antérieures, alors que d'autres sont modernes et répètent des modèles contemporains ${ }^{54}$. La question du décor nous échappe en grande partie pour l'époque donnée, mais il est assuré que certaines

52. On peut ici penser à Henri Perregaux dont la production néogothique s'explique en grande partie par la nature du commanditaire (l'église catholique) et provoque un changement majeur de manière chez cet artiste (P. Bissegger, D'ivoire et de marbre).

53. Nous avons abordé cette question dans un article récent: D. Lüthi, «Espace domestique / espaces des domestiques: autour de la chambre de bonne».

54. Comme aux Fleurettes et au Mont-d'Or où le plan des villas et des appartements - certes très simple - semble faire référence au recueil de C. Daly, L'architecture privée au XIX siècle sous Napoléon III, volume 2 en particulier. 
parties de l'immeuble, comme la cage d'escaliers, cherchent à se distinguer par un apparat spécifique et individuel, parfois même dans l'habitat ouvrier ${ }^{55}$. La façade est en revanche plus facile à appréhender et là aussi, une standardisation est palpable. Vers 1860, le style néorenaissance en vogue alors - il est à l'honneur à Paris autant qu'à Berlin - succède au néoclassicisme sobre de la période précédente. Il n'est guère d'immeuble d'un certain standing qui ne se pare de chaînes ou de pilastres d'angles, de fenêtres rectangulaires sommées d'une corniche à mascaron, de balcons filants à garde-corps de fonte. Le quasi-monopole de quelques ateliers sur le marché ${ }^{56}$ dont l'expression architecturale est assez similaire - à tel point qu'il est impossible de leur attribuer les édifices sur de simples critères stylistiques - donne à la ville du troisième quart du siècle une unité frappante. Ce phénomène n'est cependant pas propre à Lausanne et se retrouve à Genève ou à Berne par exemple. Par contraste, la sobriété de l'architecture ouvrière ne peut manquer de frapper: on a vu que dans plusieurs cas, on y retrouve des formes un peu vieillottes, néoclassiques, cherchant moins l'effet que le résultat. Mais cette indigence n'est pas qu'une question d'économie: elle est aussi affaire de morale.

Sans doute, l'élément le plus spécifique de l'arrivée des sociétés immobilières sur le marché est la "séparation" du propriétaire et de son bâtiment. L'édifice construit n'a plus pour vocation première la résidence de l'investisseur et cette rupture révèle, à notre sens, un changement de paradigme. Cet éloignement de l'objet architectural du maître de l'ouvrage implique un positionnement tout à fait différent dans le processus de la commande où les questions financières priment sans aucun doute sur les questions formelles. Comme il a été dit, le rôle de l'architecte s'en trouve valorisé car c'est sur lui que repose désormais la tâche de résoudre la formule complexe de l'adéquation entre la forme et le client potentiel. Par la place grandissante que prennent les édifices dus aux sociétés immobilières dans le paysage urbain d'alors, le rôle de ces sociétés devient prépondérant face à des autorités bien peu préoccupées par les questions d'urbanisme. Grâce à leurs initiatives privées, elles marquent

55. Voir F. Hoffmann et al., Escaliers. Décors et architecture des cages d'escaliers des immeubles d'habitation de Suisse romande 1890-1915.

56. Hormis Joël, Conod et Wanner, il faut citer Charles Mauerhoffer et Louis Bezencenet à qui l'on doit les ensembles de Georgette et de la rue du Midi, voir C. Schmutz, Louis Bezencenet (1843-1922): architecte à Lausanne. 
de leur empreinte la ville durant un temps. On est tenté de voir une reprise en main par ces sociétés bourgeoises du privilège régalien en matière d'architecture. A défaut d'un pouvoir central intéressé par la construction et l'urbanisme, c'est le domaine privé qui, via des sociétés capitalistes, endosse le rôle du prince. De la place des Vosges à l'avenue du Mont-d'Or, il n'y aurait donc qu'un pas? Si la comparaison est sans doute un peu forcée, elle a au moins le mérite de souligner à quel point l'architecture demeure un instrument du pouvoir pour celui qui construit. Seule la nature de ce pouvoir change avec les siècles.

Dave LüTHI

Section d'histoire de l'art, Université de Lausanne 
ANNEXE

\section{COMPOSITION DE QUELQUES SOCIÉTÉS IMMOBILIÈRES LAUSANNOISES DES ANNÉES 1860-1870}

\begin{tabular}{|c|c|c|c|c|c|}
\hline $\begin{array}{l}\text { Titre et date } \\
\text { de formation }\end{array}$ & Notables & Constructeurs & $\begin{array}{l}\text { Banquiers, } \\
\text { commerçants }\end{array}$ & Politiciens & Autres \\
\hline $\begin{array}{l}\text { Société } \\
\text { immobilière } \\
\text { lausannoise } \\
1860\end{array}$ & $\begin{array}{l}\text { Ch. Reneveier, } \\
\text { avocat } \\
\text { G. Perdonnet }\end{array}$ & $\begin{array}{l}\text { Louis Joël, } \\
\text { architecte }\end{array}$ & $\begin{array}{l}\text { F. Clavel, } \\
\text { banquier } \\
\text { S. Boiceau, } \\
\text { bureau du gaz }\end{array}$ & & $\begin{array}{l}\text { Francillon- } \\
\text { Agassiz } \\
\text { Ph. Ogay }\end{array}$ \\
\hline $\begin{array}{l}\text { Société de } \\
\text { construction } \\
\text { de Lausanne } \\
1861\end{array}$ & $\begin{array}{l}\text { Xavier } \\
\text { Gottofrey, } \\
\text { président, ancien } \\
\text { juge cantonal } \\
\text { Henri Bippert, } \\
\text { juge cantonal } \\
\text { (dès } 1860 \text { ) }\end{array}$ & $\begin{array}{l}\text { Ernest } \\
\text { Dapples, } \\
\text { ingénieur }\end{array}$ & $\begin{array}{l}\text { Charles Carrard, } \\
\text { banquier (dès } \\
1860 \text { ) }\end{array}$ & $\begin{array}{l}\text { Sigismond } \\
\text { Charrière de } \\
\text { Sévery, député } \\
\text { Edouard } \\
\text { Dapples, } \\
\text { député et } \\
\text { syndic (date } \\
\text { inconnue)* }\end{array}$ & \\
\hline $\begin{array}{l}\text { Société de } \\
\text { construction } \\
\text { de Lausanne } \\
\text { (comité en } \\
\text { 1896) }\end{array}$ & $\begin{array}{l}\text { Henri Bippert, } \\
\text { ancien juge } \\
\text { cantonal }\end{array}$ & $\begin{array}{l}\text { Jules } \\
\text { Duvillard, } \\
\text { ingénieur (dès } \\
1881 \text { ) } \\
\text { Alphonse } \\
\text { Vautier, } \\
\text { ingénieur (dès } \\
1886 \text { ) }\end{array}$ & $\begin{array}{l}\text { Charles Carrard, } \\
\text { banquier }\end{array}$ & & $\begin{array}{l}\text { William } \\
\text { Charrière de } \\
\text { Sévery } \\
\text { (dès 1877) } \\
\text { Théodore } \\
\text { Bergier, notaire } \\
\text { (dès 1878) }\end{array}$ \\
\hline $\begin{array}{l}\text { Association } \\
\text { coopérative } \\
\text { immobilière } \\
\text { de Lausanne } \\
1869\end{array}$ & $\begin{array}{l}\text { Charles Boiceau, } \\
\text { avocat } \\
\text { Bury, juge } \\
\text { cantonal } \\
\text { Paul Vulliet, } \\
\text { professeur }\end{array}$ & $\begin{array}{l}\text { Georges } \\
\text { Rouge, } \\
\text { architecte }\end{array}$ & $\begin{array}{l}\text { Juat-Sessler, } \\
\text { négociant }\end{array}$ & & $\begin{array}{l}\text { E. Ruchonnet, } \\
\text { major fédéral } \\
\text { Jules Brun }\end{array}$ \\
\hline
\end{tabular}

* ACV, P Curchod (Dapples) 28. Voir plus haut l'article de G. Prod'hom. 


\begin{tabular}{|l|l|l|l|l|l|}
\hline $\begin{array}{l}\text { Titre et date } \\
\text { de formation }\end{array}$ & \multicolumn{1}{|c|}{ Notables } & Constructeurs & \multicolumn{1}{|c|}{$\begin{array}{c}\text { Banquiers, } \\
\text { commerçants }\end{array}$} & Politiciens & \multicolumn{1}{|c|}{ Autres } \\
\hline $\begin{array}{l}\text { Société } \\
\text { foncière des } \\
\text { Boulevards } \\
\mathbf{1 8 7 2}\end{array}$ & $\begin{array}{l}\text { Ernest Roguin, } \\
\text { avocat }\end{array}$ & & $\begin{array}{l}\text { Adrien Mercier, } \\
\text { négociant }\end{array}$ & & $\begin{array}{l}\text { Jule Koch } \\
\text { Gustave } \\
\text { Auberjonois, } \\
\text { rentier } \\
\text { Dutoit }\end{array}$ \\
\hline $\begin{array}{l}\text { Société de la } \\
\text { rue du Jura } \\
\mathbf{1 8 7 5}\end{array}$ & J. Piot, notaire & $\begin{array}{l}\text { Jules Tarin, } \\
\text { menuisier }\end{array}$ & $\begin{array}{l}\text { L. Peitrequin, } \\
\text { cafetier } \\
\text { D. Jordan, } \\
\text { négociant }\end{array}$ & & $\begin{array}{l}\text { Vessaz } \\
\text { Alfred Renevier }\end{array}$ \\
\hline
\end{tabular}




\section{BIBLIOGRAPHIE}

\section{Abréviations}

ACV : Archives cantonales vaudoises

AVL: Archives de la Ville de Lausanne

\section{Sources publiées}

Archinard, Charles, Le Canton de Vaud et l'industrie, Lausanne, Ducloux, 1840.

Auteur anonyme, "Les habitations ouvrières - Isolement ou casernement", Journal de la Société vaudoise d'utilité publique, 1866, p. 59-64.

Crédit foncier vaudois. Septante-cinq ans d'activité: 1859-1934, Lausanne, Conseil d'administration du Crédit foncier vaudois, 1935.

DaLY, César, L'architecture privée au XIX ${ }^{e}$ siècle sous Napoléon III: nouvelles maisons de Paris et des environs, Paris, 1864.

Dapples, Ernest, Etude sur l'application des forces hydrauliques à l'exploitation des chemins de fer des montagnes et en particulier sur les chemins de fer pneumatiques, Lausanne, Martignier et Chavannes, 1866.

Notice sur les constructions exécutées à la Ponthaise par l'Association coopérative immobilière de Lausanne. Annexe au Rapport du Conseil d'Administration pour l'exercice 1871-1872, Lausanne, Bridel, 1872.

Recueil des lois, décrets et autres actes du Gouvernement du Canton de Vaud, et des actes de l'Assemblée fédérale et du Conseil fédéral de la Confédération suisse, XLIX, 1852.

Société de construction à Lausanne. Exposé sommaire et justificatif au jury de l'Exposition nationale de Genève, 1896, Lausanne, Vincent, 1896. 
Société foncière des Boulevards. Rapport du conseil d'administration à l'assemblée générale, exercice 1872-1873, Lausanne, Vincent, 1874.

Statuts de l'association coopérative immobilière de Lausanne, Lausanne, Vincent, 1869.

Statuts de la Société de construction à Lausanne, Lausanne, Vincent, 1886.

\section{Etudes}

Arlettaz, Gérald, Libéralisme et société dans le canton de Vaud: 18141845, (BHV 67), Lausanne, Bibliothèque historique vaudoise, 1980.

Barbey, Gilles, Gubler, Jacques et Abriani, Alberto, «Exemples d'habitations ouvrières au tournant du siècle», Das Werk, 63 (1976), p. 318-325.

Bissegger, Paul, Entre Arcadie et Panthéon: grandes demeures néoclassiques aux environs de Rolle, (BHV 121), Lausanne, Bibliothèque historique vaudoise, 2001.

-, D'ivoire et de marbre: Alexandre et Henri Perregaux ou L'âge d'or de l'architecture vaudoise, 1770-1850, (BHV 131), Lausanne, Bibliothèque historique vaudoise, 2007.

Corboz, André, "Le palais Eynard à Genève: un Design architectural en 1817 ", Genava, 23 (1975), p. 195-275.

Corthésy, Bruno, «"L'ouvrier d'aujourd'hui aime aussi le confort et les belles choses." Les quatre petites maisons ouvrières de l'Usine à gaz de Malley, Renens", in Logement ouvrier, (Cahiers d'histoire du mouvement ouvrier 25), Lausanne, Editions d'En bas, 2009, p. 41-52.

Grandjean, Marcel, Les Monuments d'art et d'histoire du canton de Vaud, t. IV, Lausanne: villages, hameaux et maisons de l'ancienne campagne lausannoise, Bâle, Birkhäuser, 1981.

Heller, Geneviève, "Propre en ordre". Habitation et vie domestique 1850-1930: l'exemple vaudois, Lausanne, Ed. d'En Bas, 1979.

Hoffmann, Fabienne, Lüthi, Dave, Maillard, Nadja, Reymond Bui, Catherine et Schmutz Nicod, Catherine, Escaliers. Décors et architecture des cages d'escaliers des immeubles d'habitation de Suisse romande 1890-1915, Lausanne, Presses polytechniques et universitaires romandes, 2006. 
INSA, inventaire suisse d'architecture 1850-1920, t. 5, Berne, 1990.

Lüthi, Dave, Le quartier du Mont-d'Or à Lausanne. Etude historique, urbanistique et architecturale, Service des bâtiments de l'Etat, section Monuments et sites, 2003-2004 (inédit).

—, "Espace domestique / espaces des domestiques: autour de la chambre de bonne", in Logement ouvrier, (Cahiers d'histoire du mouvement ouvrier 25), Lausanne, Editions d'En bas, 2009, p. 85-97.

Montserrat Farguell, Isabelle et Grandval, Virginie (dir.), Hameaux, villas et cités de Paris, Paris, Délégation à l'action artistique de la ville de Paris, 1998.

Nagel, Anne, Möhle, Martin et Meles, Brigitte, Die Kunstdenkmäler des Kantons Basel-Stadt, t. VII, Altstadt Grossbasel I, Berne, Société d'histoire de l'art en Suisse, 2006.

Pinon, Pierre, Atlas du Paris haussmannien. La ville en héritage du Second Empire à nos jours, Paris, Parigramme, 2002.

Schmutz, Catherine, Louis Bezencenet (1843-1922): architecte à Lausanne, mémoire de licence en histoire de l'art, Université de Lausanne, Faculté des Lettres, 1996.

\section{Crédits photographiques}

Fig. $1-3,5,7$ et 8 :

Dave Lüthi.

Fig. 4 et 6 :

Archives de la Ville de Lausanne. 